\author{
Y. F. Hu - H. L. Zhang - T. Cai - S. Harashima • \\ A. L. Notkins
}

\title{
The IA-2 interactome
}

Received: 15 July 2005 / Accepted: 2 August 2005 / Published online: 5 November 2005

(C) Springer-Verlag 2005

\begin{abstract}
Aims/hypothesis: Islet antigen-2 (IA-2), a major autoantigen in type 1 diabetes, is an enzymatically inactive member of the transmembrane protein tyrosine phosphatase (PTP) family. IA-2 is located in dense-core secretory vesicles and is involved in the regulation of insulin secretion. The present experiments were initiated to identify those proteins that interact with IA-2 (i.e. the IA-2 interactome) as a first step towards elucidating the mechanism(s) by which IA-2 influences insulin secretion and serves as an autoantigen. Materials and methods: To determine the proteins with which IA-2 interacts, a yeast twohybrid system was used to screen a human foetal library, and deletion mutants were used to determine the binding sites. Positive interactions were confirmed by immunoprecipitation pull-down experiments using cell lysate from transfected mammalian cell lines. Results: Six new interacting proteins were identified by this approach: mitogenactivated protein kinase-activating death domain (MADD), the MADD isoform IG20, PTP $\rho, \mathrm{PTP} \sigma$, sorting nexin 19 (SNX19) and cyclophilin A. Using a series of IA-2 deletion mutants, we identified the regions on the IA-2 molecule to which five of the interacting proteins bound. Amino acids 744-979 of IA-2 were required for the maximum binding of MADD, IG20 and SNX19, whereas amino acids $602-907$ of IA-2 were required for the maximum binding of PTP $\rho$ and PTP $\sigma$. Pull-down experiments with cell lysate from transfected mammalian cells confirmed the binding of the interacting proteins to IA-2.
\end{abstract}

\footnotetext{
Y. F. Hu $\cdot$ T. Cai $\cdot$ S. Harashima $\cdot$ A. L. Notkins $(\bowtie)$ Experimental Medicine Section,

Oral Infection and Immunity Branch,

National Institutes of Dental and Craniofacial Research,

National Institutes of Health,

Bethesda, MD 20892, USA

e-mail: anotkins@mail.nih.gov

Tel.: +1-301-4964535

Fax: +1-301-4024163

H. L. Zhang

Laboratory of Molecular Signaling and Apoptosis,

Department of Biologic and Materials Science,

School of Dentistry, University of Michigan,

Ann Arbor, MI, USA
}

Conclusions/interpretation: The IA-2 interactome, based on pull-down experiments, currently consists of 12 proteins. The identification of these interacting proteins provides clues as to how IA-2 exerts its biological functions.

Keywords Autoimmunity - Dense-core secretory vesicles · IA-2 - Insulin secretion · Interactome · Protein interaction Protein tyrosine phosphatase Type 1 diabetes $\cdot$ Yeast two-hybrid

Abbreviations aa: amino acids $\cdot \mathrm{AD}$ : activating domain . BD: binding domain - CypA: cyclophilin A .

DCV: dense-core secretory vesicles - HA: influenza virus haemagglutinin - IA-2: islet antigen-2 - IA-2ic: intracellular domain of IA-2 - MADD: mitogen-activated protein kinase-activating death domain $\cdot \mathrm{nNOS}$ : neuronal nitric oxide synthase - PX: Phox - PTP: protein tyrosine phosphatase $\cdot$ SNX19: sorting nexin 19

\section{Introduction}

Islet antigen-2 (IA-2) is a major autoantigen in type 1 diabetes [1]. About $70 \%$ of newly diagnosed type 1 patients have autoantibodies to IA-2, and these autoantibodies appear years before the development of clinical disease. Screening of various populations (first-degree relatives and school children) has shown that individuals who have autoantibodies to both IA-2 and GAD65 have an approximately $50 \%$ risk of developing type 1 diabetes within 5 years. Thus, autoantibody screening is being widely used to identify high-risk subjects for entry into therapeutic intervention trials.

Based on sequence data, IA-2 (also known as ICA512) is a member of the transmembrane protein tyrosine phosphatase (PTP) family [2]. IA-2 is 979 amino acids (aa) in length, is located on chromosome $2 \mathrm{q} 35$, and consists of an extracellular domain (aa 1-576), a transmembrane region (aa 577-600) and an intracellular domain (aa 601-979). However, IA-2 is enzymatically inactive when tested with 
known PTP substrates due to the substitution of two amino acids (Ala $911 \rightarrow$ Asp and Asp 877 $\rightarrow$ Ala) at conserved sites known to be critical for PTP activity. Site-directed mutagenesis restores enzyme activity. Immunofluoresence studies have shown that IA-2 is located in neuroendocrine cells throughout the body, including beta and alpha cells in pancreatic islets, and electron microscopic studies have demonstrated that IA-2 is an integral component of dense-core secretory vesicles (DCV) [3]. Deletion of the gene encoding IA-2 in mice results in impaired glucose-induced insulin secretion and elevated responses to glucose tolerance tests [4]. Conversely, glucose-induced insulin secretion is increased several fold in response to the overexpression of IA2 in cell lines [5]. These and other experiments show that IA-2 is a positive modulator of insulin secretion. However, the molecular mechanism(s) by which IA-2 influences insulin secretion is not known. A first step towards the elucidation of the mechanism(s) is the identification of those proteins with which IA-2 interacts. In the present experiments we used the yeast two-hybrid system to screen human foetal libraries for IA-2-interacting proteins and then confirmed the interactions by performing immunoprecipitation pull-down experiments in transfected mammalian cells.

\section{Materials and methods}

Antibodies Rabbit polyclonal IgG antibodies against influenza virus haemagglutinin (HA; Y-11) or c-Myc (A-14), and mouse monoclonal IgG antibody against c-Myc (9E10) were purchased from Santa Cruz Biotechnology (Santa Cruz, CA, USA). Mouse monoclonal IgG antibodies against $6 \times$ His tag or the binding domain (BD) of GAL4 were obtained from BD Biosciences (Palo Alto, CA, USA). Mouse monoclonal IgG antibody against HA (12CA5) came from Roche (Basel, Switzerland). Rabbit polyclonal IgG antibody to the $\mathrm{C}$-terminal of mitogen-activated protein kinase-activating death domain (MADD; aa 1,570-1,588) was obtained from EMD Biosciences (San Diego, CA, USA), and rabbit polyclonal IgG antibody against human cyclophilin A (CypA) was obtained from Upstate Biotechnology (Lake Placid, NY, USA). A Western-Light detection kit was purchased from Applied Biosystems (Foster City, CA, USA). Protein G beads were from Sigma-Aldrich (St Louis, MO, USA), and LipofectAmine Plus transfection reagents were from Invitrogen (Carlsbad, CA, USA).

Yeast two-hybrid screening The Matchmaker Two-Hybrid System 3 was purchased from BD Biosciences. Use of the yeast strain AH109, which contains three reporter genes (ADE2, HIS3 and MEL1) that are under the control of distinct GAL4 upstream activating sequences and TATA boxes, tends to eliminate most technical false positives. The bait cDNA, encoding the intracellular domain of IA-2 (IA-2ic; aa 602-979), was generated by PCR and inserted in-frame with the GAL4 DNA binding domain (BD) in the high-expression vector pGBKT7 or the low-expression vector pGBT9 (BD Biosciences). The successful expres- sion of the BD-IA-2ic fusion protein in yeast strain AH109 was verified by Western blotting using anti-GAL4-BD monoclonal antibody. The screening was carried out according to the manufacturer's protocol, as previously reported [6]. Briefly, AH109 cells bearing pGBT9-IA-2ic were transformed with the human foetal brain cDNA library in pACT2 vector (BD Biosciences). The transformants containing interacting proteins were selected on synthetic dropout medium (lacking the four nutrients adenine, histidine, leucine and tryptophan, and containing $\mathrm{X}$ - $\alpha$-gal medium). The plates were searched for colonies that survived as a result of the expression of HIS3, ADE2 and MEL1 (i.e. blue colonies). The surviving blue clones were amplified, and plasmids were isolated and sequenced.

Mutagenesis IA-2 carboxyl-terminal deletion mutants were generated by PCR or restriction enzyme digestion and were subcloned into pGBKT7. MADD (aa 1,356$1,588)$ without the carboxyl-terminal death domain was amplified by PCR and inserted into pGADT7 (BD Biosciences). Two sorting nexin 19 (SNX19) mutants (aa 494-992 with the Phox [PX] domain and aa 676-992 without the PX domain) were constructed from pACT2SNX19 (aa 258-992) by restriction enzyme digestion.

$\alpha$-Galactosidase quantitative assay The assay was performed as previously reported [6]. In brief, AH109 yeast cells carrying BD-IA-2 mutants were mated with Y187 yeast cells carrying proteins that interact with IA-2, and then plated on synthetic dropout medium (i.e. lacking leucine and tryptophan) to produce the BD-fusion and the AD-fusion proteins. Single clones were inoculated and grown in liquid culture medium overnight at $30^{\circ} \mathrm{C}$, and then optical densities $\left(\mathrm{OD}_{600}\right)$ were measured. The concentration of $\alpha$-galactosidase $(\mathrm{mU} / \mathrm{ml})$ secreted into the culture medium was determined by adding 4-nitrophenyl- $\alpha$-D-galactoside, a substrate of the enzyme (Sigma-Aldrich).

Immunoprecipitation in mammalian cells pCMV-Myc-IA2ic (aa 602-979) and pCMV-Tag (Flag)-IA-2ic (aa 602979) were constructed by excising IA-2ic from pGBKT7IA-2ic and inserting it into vector pCMV-Myc (BD Biosciences) or pCMV-Tag (Flag) (Stratagene, La Jolla, CA). We generated pCMV-HA-MADD (aa 1,288-1,588) by excising the MADD fragment from plasmid pACT2MADD and inserting it into pCMV-HA (BD Biosciences). pCMV-Myc-SNX (aa 258-992), pCMV-Myc-PTP $\rho$ (aa 857-1,400) and pCMV-Myc-PTP $\sigma$ (aa 1697-1,948) were generated by excising fragments from pACT2-SNX, pACT2-PTP $\rho$ or pACT2-PTP $\sigma$ and inserting them into vector pCMV-Myc. The full-length cDNA of the gene encoding human CypA was cloned into the pcDNA3.1/GS vector (Invitrogen) with the $6 \times$ His tag as a fusion protein. The pcDNA3.1/His-MADD and pcDNA3.1/ His-IG20 (an isoform of MADD) plasmids were kindly provided by B. S. Prabhakar (Department of Microbiology and Immunology, University of Illinois, Chicago, IL, USA) [7].

HEK293 cells were cultured in DMEM media with $10 \%$ foetal bovine serum and $100 \mathrm{U}$ of penicillin-streptomycin 
at $37^{\circ} \mathrm{C}, 5 \% \mathrm{CO}_{2}$, in a humidified incubator. Cells in 10 $\mathrm{cm}$ dishes were co-transfected with $8 \mu \mathrm{g}$ of a pair of vectors containing cDNA of the gene for IA-2 or the gene of the interacting protein, or $8 \mu \mathrm{g}$ of a pair of control vectors, using LipofectAmine Plus reagent according to the manufacturer's instructions. After $48 \mathrm{~h}$, cells were harvested and placed in lysis buffer (PBS, pH 7.4, containing $1 \%$ Triton $\mathrm{X}-100,0.1 \mathrm{mmol} / \mathrm{l}$ EDTA and complete proteinase inhibitor cocktail) (Roche). Samples $(500 \mu \mathrm{g})$ of total protein from the cell extracts were precleared by incubation with Protein $\mathrm{G}$ beads for $30 \mathrm{~min}$ at $4^{\circ} \mathrm{C}$. The samples were then immunoprecipitated with $1.0 \mu \mathrm{g}$ of antibody for $2 \mathrm{~h}$ at $4^{\circ} \mathrm{C}, 30 \mu \mathrm{l}$ of $50 \%$ Protein $\mathrm{G}$ bead slurry was added, and then the reaction mixture was incubated for a further $2 \mathrm{~h}$ at $4^{\circ} \mathrm{C}$. The beads were washed four times with $500 \mu \mathrm{l}$ of cold lysis buffer, suspended in $50 \mu \mathrm{l}$ of $1 \times$ SDS loading buffer, and boiled for $10 \mathrm{~min}$. The proteins were separated by electrophoresis through a 4 $12 \%$ SDS-PAGE gel and analysed by Western blotting.

\section{Results}

Identification of IA-2-interacting proteins and mapping of the IA-2 binding sites The yeast two-hybrid system was used to identify proteins that interact with IA-2. Of the approximately 4.5 million transformants that were screened, nine colonies survived the multiple screening steps. Sequencing revealed three MADD clones (aa 1,288-1,588, aa 1,347-1,588, aa 1,350-1,588; GenBank accession number AAB57735), three PTP $\sigma$ clones (aa 857-1,400; GenBank accession number NP 002841), one PTP $\rho$ clone (aa 1,6971,948; GenBank accession number XP_012936), one
SNX19 clone (aa 258-992; GenBank accession number NP_055573) and one CypA clone (aa 1-164; GenBank accession number P05092). To determine the regions of IA-2 to which these proteins bind, a series of IA-2 deletion mutants were prepared. The binding of MADD, PTP $\rho$, PTP $\sigma$ and SNX19 to each of the IA-2 deletion mutants was evaluated by growth on selective medium plates and by quantification of $\alpha$-galactosidase activity. As seen in Table 1, a fragment of MADD (aa 1,356-1,588) that did not contain its death domain [8] bound to IA-2ic (aa 602 979). Further studies showed that the critical binding region was between amino acids 744 and 979. Deletion of the Cterminal of IA-2 (fragments consisting of aa 602-907, 693917, 693-923 and 693-942) abolished the interaction between IA-2 and MADD, as did deletion of 60 amino acids from the N-terminal part (aa 804-979) of the best IA-2interacting mutant (aa 744-979). A similar (but weaker) binding profile to IA-2 was found with SNX19. Only SNX19 containing the PX domain (aa 494-992) bound to IA-2. SNX19 without the PX domain (aa 676-992) did not bind to IA-2 (data not shown). In contrast to MADD and SNX19, PTP $\rho$ and PTP $\sigma$ did not require the PTP core domain of IA-2 for binding. The best binding occurred with the IA-2 mutant that consisted of amino acids 602-907 of the full-length protein. Deletion of 91 amino acids from the $\mathrm{N}$-terminal of this mutant substantially reduced binding activity, especially that determined by $\alpha$-galactosidase activity. PTP $\rho$ (aa 857-1,400) contained both D1 (aa 847-1104) and D2 (aa 1,134-1,397) domains. PTP $\sigma$ (aa $1,697-1,948$ ) contained only the D2 domain (aa 1,8361,938). The binding of PTP $\rho$ to IA-2 was stronger than the binding of PTP $\sigma$ to IA- 2 , as measured by $\alpha$-galactosidase activity.

Table 1 Regions of IA-2 to which interacting proteins bind

\begin{tabular}{|c|c|c|c|c|c|c|c|c|c|c|}
\hline \multicolumn{3}{|c|}{$\mathrm{IA}-2$} & \multicolumn{8}{|c|}{ IA-2-interacting proteins } \\
\hline $\begin{array}{c}\text { SP } \\
1-26 \\
\end{array}$ & $\begin{array}{c}\text { TM } \\
577-600 \\
\end{array}$ & $\begin{array}{l}\text { PTP Core } \\
907-917\end{array}$ & \multicolumn{2}{|c|}{$\begin{array}{c}\text { MADD } \\
\text { (aa } 1356-1588 \text { ) }\end{array}$} & \multicolumn{2}{|c|}{$\begin{array}{c}\text { SNX19 } \\
\text { (aa 494-992) }\end{array}$} & \multicolumn{2}{|c|}{$\begin{array}{c}\text { PTP } \rho \\
\text { (aa } 857-1400 \text { ) }\end{array}$} & \multicolumn{2}{|c|}{$\begin{array}{c}\text { PTP } \sigma \\
\text { (aa 1697-1948) }\end{array}$} \\
\hline 1 & 500 & 979 & Plates & $\alpha-G a l$ & Plates & $\alpha-G a l$ & Plates & $\alpha-\mathrm{Gal}$ & Plates & $\alpha-G a l$ \\
\hline IA-2 (602-979) & & & ++ & 10.0 & + & 3.0 & +++ & 36.9 & + & 5.6 \\
\hline IA-2 (602-706) & & & - & 1.2 & - & 0.7 & - & 0.8 & - & 0.7 \\
\hline IA-2 (602-907) & & & - & 0.8 & - & 0.9 & +++ & 79.9 & +++ & 29.3 \\
\hline IA-2 (693-979) & & & ++ & 10.4 & + & 3.1 & +++ & 33.4 & + & 1.9 \\
\hline IA-2 (693-917) & & & - & 0.6 & - & 0.8 & +++ & 42.3 & + & 9.1 \\
\hline IA-2 (693-923) & & & - & 0.7 & - & 0.8 & ++ & 14.7 & ++ & 10.1 \\
\hline IA-2 (693-942) & & & - & 0.8 & - & 0.9 & ++ & 13.6 & \pm & 1.4 \\
\hline IA-2 (744-979) & & & +++ & 35.5 & + & 6.4 & - & 0.8 & - & 0.8 \\
\hline IA-2 (804-979) & & $T$ & - & 1.2 & - & 0.8 & - & 0.9 & - & 0.6 \\
\hline
\end{tabular}

The IA-2ic deletion mutants shown in the left column were used as bait to screen the binding of MADD, SNX19, PTP $\rho$ and PTP $\sigma$ by the yeast two-hybrid system. Matings that survived on plates (Plates column) (blue colonies) with synthetic dropout medium were scored according to the speed of growth. Colonies that appeared blue 2, 3 and 4 days after plating were scored as $(+++),(++)$ and $(+)$ respectively, and at the end of 1 week as $( \pm)$. Plates on which colonies failed to appear after 7 days were scored as negative $(-)$. Separately, in a liquid selective medium assay, the extent of interaction was quantified by the activity of the reporter gene encoding $\alpha$-galactosidase (mU/ml) $(\alpha$ Gal column). SP signal peptide, TM transmembrane region, PTP core protein tyrosine phosphatase core domain 
a

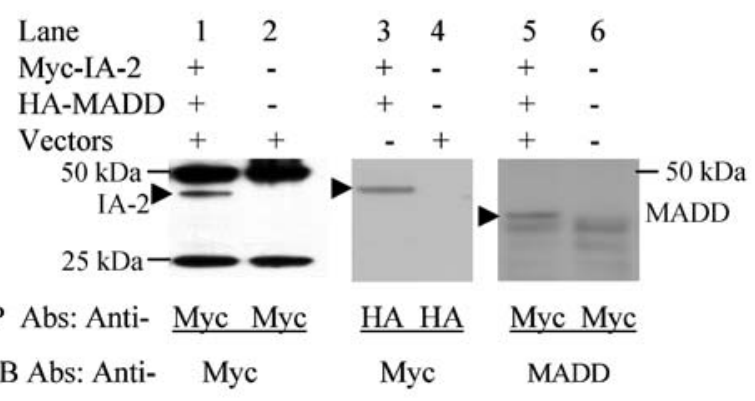

c

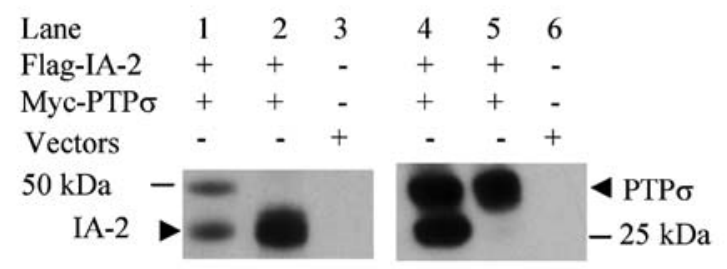

IP Abs: Anti- Flag Myc Myc Flag Myc Myc

WB Abs: Anti- Flag Myc

e

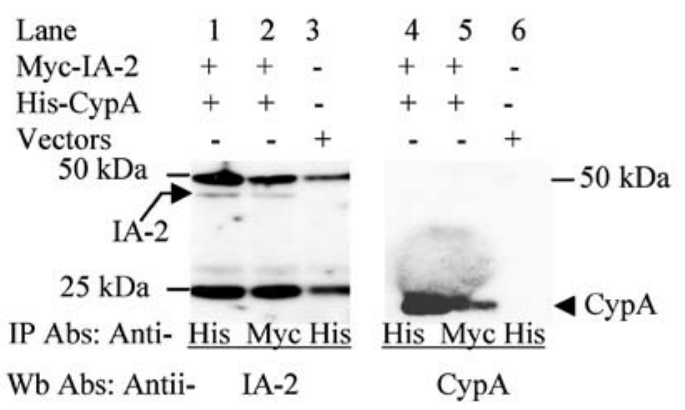

b

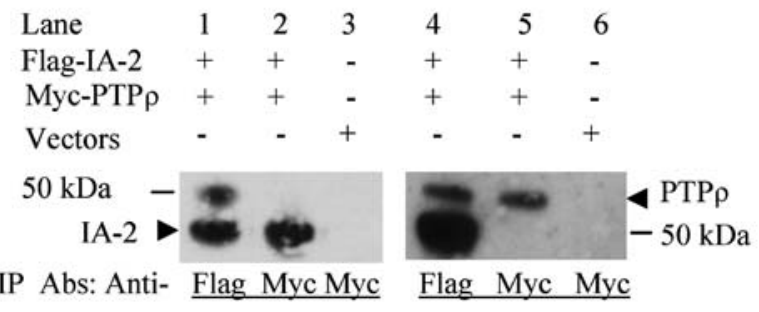

WB Abs: Anti- Flag Myc

d

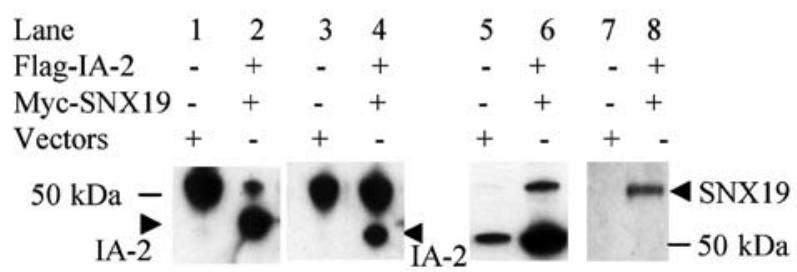

IP Abs: Anti- $\underline{\text { Flag }} \underline{\text { Myc }} \underline{\text { Flag }} \underline{\text { Myc }}$

WB Abs:Anti- Flag Myc

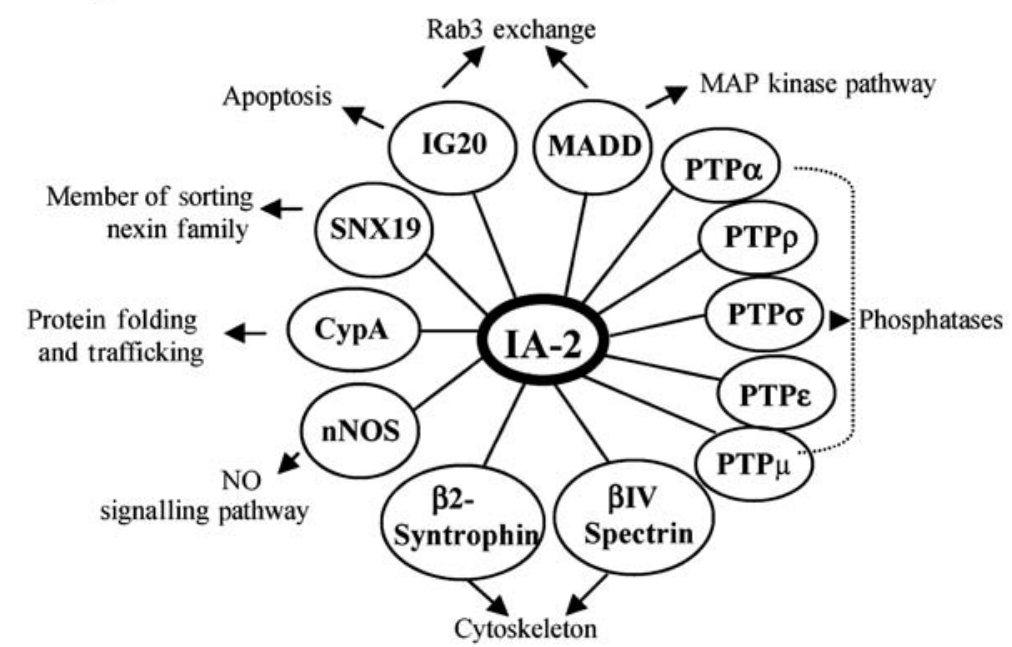

Fig. 1 Co-immunoprecipitation in mammalian cells of IA-2 and interacting proteins. Myc-tagged or Flag-tagged IA-2ic (aa 602-979, $43 \mathrm{kDa}$ ) was co-transfected with HA-tagged MADD (aa 1,2881,588, $35 \mathrm{kDa}$ ), Myc-tagged PTP $\rho$ (aa 857-1,400, $62 \mathrm{kDa}$ ), Myctagged PTP $(1,697-1,948,29 \mathrm{kDa})$, Myc-tagged SNX19 (aa 258992, $80 \mathrm{kDa}$ ) or His-tagged CypA (full length, $20 \mathrm{kDa}$ ) in HEK293 cells. Cells co-transfected with empty vectors served as controls. a immunoprecipitation of HA-MADD with anti-HA antibody brought down IA-2 (lane 3), and immunoprecipitation of Myc-IA-2 with anti-Myc brought down MADD (lane 5). Immunoprecipitation of Myc-PTP $\rho$ (b) or Myc-PTP $\sigma$ (c) with anti-Myc antibody brought down IA-2 (lane 2), and immunoprecipitation of Flag-IA-2 with antiFlag antibody brought down PTP $\rho$ or PTPo (b, c lane 4). d

Immunoprecipitation of Myc-SNX19 with anti-Myc antibody brought down IA-2 (lane 4), and immunoprecipitation of Flag-IA2 with anti-Flag antibody brought down SNX19 (lane 6). e Immunoprecipitation of His-CypA with anti-His antibody brought down IA-2 (lane 1), and immunoprecipitation of Myc-IA-2 with anti-Myc antibody brought down CypA (lane 5). In a-e, IgG heavy chains are seen at $50 \mathrm{kDa}$ and $\mathrm{IgG}$ light chains at $25 \mathrm{kDa}$. f The proteins of the IA-2 interactome identified by pull-down experiments in the current study (CypA, SNX19, IG20, MADD, PTP $\rho$ and PTP $\sigma)$ and from the literature $(\mathrm{PTP} \alpha, \mathrm{PTP} \varepsilon, \mathrm{PTP} \mu, \beta \mathrm{IV}$ spectrin, $\beta 2$ syntrophin and nNOS). Some of the known functions of the interacting proteins are indicated. IP $A b s$ antibodies used for immunoprecipitation, WB $A b s$ antibodies used for Western blotting 
Co-immunoprecipitation of IA-2-interacting proteins with IA-2 To determine whether the interaction demonstrated by the yeast two-hybrid system occurred in mammalian cells, pull-down experiments were performed. Mammalian cells (HEK293) were co-transfected with IA-2 and one of the interacting proteins (MADD, IG20, SNX19, PTP $\rho$, PTP $\sigma$ or CypA). Lysates were prepared, immunoprecipitated with antibody to one of the transfected proteins and then screened for the interacting protein with antibody to that protein by Western blotting. Figure 1a shows that in cells transfected with both IA-2 and MADD, immunoprecipitation of HA-MADD with anti-HA pulled down IA-2 (lane 3 ) and that immunoprecipitation of Myc-IA-2 with antiMyc pulled down MADD (lane 5). Similar results were obtained with IG20 [7], the largest isoform of MADD, but binding to the N-terminal of MADD did not occur (not shown). In cells transfected with both IA-2 and either PTP $\rho$ or PTP $\sigma$ (Fig. 1b,c) immunoprecipitation of MycPTP $\rho$ or Myc-PTP $\sigma$ with anti-Myc pulled down IA-2 (lane 2), and immunoprecipitation of Flag-IA-2 with antiFlag pulled down PTP $\rho$ and РTPб (lane 4). Similarly, SNX19 (lane 6 in Fig. 1d) and CypA (lane 5 in Fig. 1e) were pulled down by antibody to IA-2, and antibody to SNX19 or to CypA pulled down IA-2 (lane 4 in Fig. 1d or lane 1 in Fig. 1e). Cyclosporin A, which is known to bind to CypA [9], completely blocked the binding of IA-2 to CypA (data not shown).

\section{Discussion}

Although of enormous value, the yeast two-hybrid system often fails to identify proteins that interact with one another. In our experiments, we screened two libraries a total of four times. Only one of the proteins, MADD, was positively identified on three of the screenings, and another protein, $\mathrm{PTP} \sigma$, was positively identified on two of the screenings. The other four interacting proteins were positively identified on only one of the screenings. In this context it is of interest that proteins previously identified as IA-2-interacting proteins by yeast two-hybrid screening ( $\beta 2$-syntrophin, $\beta I V$ spectrin and neuronal nitric oxide synthase [nNOS]), [10], were not detected in any of our screenings. Indeed, three other proteins previously identified as IA-2-interacting proteins were not detected in our screening experiments, but these proteins were specifically selected for study because of the known heterodimerisation of PTP proteins [11].

Another problem with the yeast two-hybrid system is the not infrequent false positive interactions. For this reason we confirmed all our yeast two-hybrid findings with pulldown experiments in transfected mammalian cells [12]. In addition, using non-transfected Min-6 cells, we demonstrated the endogenous pull-down of MADD with IA-2 and SNX19 with IA-2 (not shown). The value of identifying IA-2-interacting proteins is that they provide clues as to the possible molecular mechanism(s) by which IA-2 might operate as a regulator or modifier of glucose-induced and basal insulin secretion. For example, IA-2 binds to human
MADD. The mouse homologue of MADD, Rab3GEP, upregulates Rab3 exchange [13]. Rab3 is known to be involved in the secretory machinery of beta cells and acts at several different levels in regulated secretion. In this context, we found that high levels of IA-2, MADD and Rab3 A are produced in mouse pancreatic beta cells, and confocal microscopy showed that IA-2 co-localises with MADD (not shown). The PTPs are known to be involved in one or more steps of the DCV secretory pathways. The demonstration that IA-2 can bind to at least five different PTPs and can, in at least one case, inhibit enzymatic activity [11], points to a second possible mechanism by which IA-2 might modulate secretion. The function of SNX19 has not been determined, but its PX domain has the capacity to bind to phospholipids [14]. Furthermore, the PX domain appears to be required for the binding of IA-2. Since some members of the SNX family are known to function in the endocytosis of plasma-membrane receptors and/or the trafficking of proteins from one membrane compartment to another, it is possible that IA-2 might modulate DCV trafficking and secretion through its interaction with SNX19. Similarly, the binding of IA-2 to the cytoskeleton proteins $\beta 2$-syntrophin and $\beta$ IV spectrin could affect trafficking of DCV, and the binding of IA-2 to the PDZ domain of nNOS might affect NO signalling involved in the secretion of hormones [10]. CypA has a variety of cellular (e.g. protein folding and trafficking) and immune-related functions [9], but precisely how its interaction with IA-2 influences these functions remains to be determined.

The IA-2 interactome currently consists of 12 interacting proteins, all of which have been confirmed by pull-down experiments (Fig. 1f). Almost certainly, upon further screening, additional members of the IA-2 interactome will be found. In Caenorhabitditis elegans, a single protein has been shown to interact with dozens of different proteins and, in turn, each of these proteins can interact with still other proteins to form a network of interactions [12]. In terms of IA-2, the challenge now is to determine the proteins and the mechanism(s) involved in DCV secretion. Equally important is the role, if any, that these interacting proteins play in triggering the autoimmune response to IA-2.

Acknowledgements This research was supported by the Intramural Research Program of the National Institute of Dental and Craniofacial Research, the National Institutes of Health.

\section{References}

1. Notkins AL (2002) Immunologic and genetic factors in type 1 diabetes. J Biol Chem 277:43545-43548

2. Lan MS, Lu J, Goto Y, Notkins AL (1994) Molecular cloning and identification of a receptor-type protein tyrosine phosphatase, IA-2, from human insulinoma. DNA Cell Biol 13:505514

3. Solimena M, Dirkx R Jr, Hermel JM et al (1996) ICA 512, an autoantigen of type I diabetes, is an intrinsic membrane protein of neurosecretory granules. EMBO J 15:2102-2114 
4. Saeki K, Zhu M, Kubosaki A, Xie J, Lan MS, Notkins AL (2002) Targeted disruption of the protein tyrosine phosphataselike molecule IA-2 results in alterations in glucose tolerance tests and insulin secretion. Diabetes 51:1842-1850

5. Harashima S, Clark A, Christie MR, Notkins AL (2005) The dense core transmembrane vesicle protein IA-2 is a regulator of vesicle number and insulin secretion. Proc Natl Acad Sci U S A 102:8704-8709

6. Hu Y, Fang X, Dunham SM, Prada C, Stachowiak EK, Stachowiak MK (2004) 90-kDa ribosomal S6 kinase is a direct target for the nuclear fibroblast growth factor receptor 1 (FGFR1): role in FGFR1 signaling. J Biol Chem 279:2932529335

7. Al-Zoubi AM, Efimova EV, Kaithamana S et al (2001) Contrasting effects of IG20 and its splice isoforms, MADD and DENN-SV, on tumor necrosis factor alpha-induced apoptosis and activation of caspase- 8 and -3 . J Biol Chem 276:47202-47211

8. Schievella AR, Chen JH, Graham JR, Lin LL (1997) MADD, a novel death domain protein that interacts with the type 1 tumor necrosis factor receptor and activates mitogen-activated protein kinase. J Biol Chem 272:12069-12075
9. Colgan J, Asmal M, Neagu M et al (2004) Cyclophilin A regulates TCR signal strength in CD4+ T cells via a prolinedirected conformational switch in Itk. Immunity 21:189-201

10. Ort T, Maksimova E, Dirkx R et al (2002) The receptor tyrosine phosphatase-like protein ICA512 binds the PDZ domains of beta2-syntrophin and nNOS in pancreatic beta-cells. Eur J Cell Biol 79:621-630

11. Gross S, Blanchetot C, Schepens J et al (2002) Multimerization of the protein-tyrosine phosphatase (PTP)-like insulin-dependent diabetes mellitus autoantigens IA-2 and IA- $2 \beta$ with receptor PTPs (RPTPs). Inhibition of RPTP $\alpha$ enzymatic activity. J Biol Chem 277:48139-48145

12. Li S, Armstrong CM, Bertin N et al (2004) A map of the interactome network of the metazoan $C$. elegans. Science 303:540-543

13. Yamaguchi K, Tanaka M, Mizoguchi A et al (2002) A GDP/ GTP exchange protein for the Rab3 small G protein family upregulates a postdocking step of synaptic exocytosis in central synapses. Proc Natl Acad Sci USA 99:14536-14541

14. Worby CA, Dixon JE (2002) Sorting out the cellular functions of sorting nexins. Nat Rev Mol Cell Biol 3:919-931 